\title{
Influence of Weed Management Practices on Uptake of Nutrients by Kharif Sorghum [Sorghum bicolor (L.) Moench] and its Effect on Soil Fertility Status
}

\section{S. S. Vinayaka ${ }^{1}$, D. Krishnamurthy ${ }^{1}$, A. S. Channabasavanna ${ }^{2}$, Y. M. Ramesha ${ }^{3}$ and Manjunath Bhanuvalli ${ }^{4}$}

\author{
${ }^{1}$ Department of Agronomy, AICRP on Sorghum, ARS, Hagari, India \\ ${ }^{2}$ Department of Agronomy, Directorate of Research, UAS, Raichur, India \\ ${ }^{3}$ Department of Agronomy, ARS, Dhadesugur, India \\ ${ }^{4}$ Department of Soil Science, Agricultural Extension Education Centre, \\ Hadagali, University of Agricultural Sciences, Raichur-584 104, India
}

*Corresponding author

\section{A B S T R A C T}

\section{Keywords}

Polebean, Tillage, Nutrient uptake and microbial population

\section{Article Info}

Accepted: 04 September 2019 Available Online: 10 October 2019
A field experiment was conducted at Agricultural Research Station, Hagari, Ballari during Kharif season of 2018on deep black soil. The experiment was laid out in randomized block design and repeated thrice with twelve treatments. Significantly higher uptake of nitrogen, phosphorus and potassium $\left(219,56.5\right.$ and $\left.147.4 \mathrm{~kg} \mathrm{ha}^{-1}\right)$ was recorded with atrazine $50 \mathrm{WP}$ @ 0.50 a.i. kg ha ${ }^{-1} \mathrm{fb} 2,4-\mathrm{D}$ Ethyl Ester 38 EC @ 0.90 a.i. $\mathrm{kg} \mathrm{ha}^{-1}$ as PoE at 30 DAS and it was on par with hand weeding at 20 DAS and 1 IC at 40 DAS $(215,55.2$, and $145.7 \mathrm{~kg}$ $\mathrm{ha}^{-1}$, respectively) and 2,4-D Ethyl Ester 38 EC @ $0.90 \mathrm{~kg}$ a.i. ha ${ }^{-1}$ as PoE at 30 DAS fb 1 IC at 40 DAS $\left(211,54.9\right.$, and $143.7 \mathrm{~kg} \mathrm{ha}^{-1}$, respectively. Nitrogen, phosphorous and potassium availability in the soil was significantly higher with weed free (IC at $20 \& 40$ DAS and HW at 30 DAS) $\left(194,47.6\right.$, and $219.9 \mathrm{~kg} \mathrm{ha}^{-1}$, respectively) treatment. Among other weed management practices, sequential pre-emergence application of atrazine $50 \mathrm{WP}$ @ $0.50 \mathrm{~kg}$ a.i. $\mathrm{ha}^{-1} \mathrm{fb} 2,4-\mathrm{D}$ Ethyl Ester $38 \mathrm{EC} @ 0.90 \mathrm{~kg}$ a.i. $\mathrm{ha}^{-1}$ as PoE at $30 \mathrm{DAS}$ recoded significantly higher availability of nitrogen, phosphorous and potassium in the soil (177, 42.2, and $213.8 \mathrm{~kg} \mathrm{ha}^{-1}$, respectively) and it was on par with hand weeding at 20 DAS and 1 IC at 40 DAS $\left(173,41.7\right.$, and $212.1 \mathrm{~kg} \mathrm{ha}^{-1}$, respectively) and 2,4-D Ethyl Ester 38 EC @ $0.90 \mathrm{~kg}$ a.i. $\mathrm{ha}^{-1}$ as PoE at 30 DAS fb 1 IC at 40 DAS (170, 39.9, and 211.9 $\mathrm{kg} \mathrm{ha}^{-1}$, respectively).

\section{Introduction}

Sorghum is one of the important dryland crop grown in poor lands with minimum inputs and in dry conditions that tolerate to heat, salt and water-logging. Sorghum is a preferred in tropical, warm and semiarid regions of the world with high temperature and water stress 
with the threat of climate change looming large on the crop productivity, sorghum is hardy crop that plays an important role in food, feed and fodder security in dryland economy (Paterson et al., 2009). Sorghum is a smart crop capable of providing raw material for the making of starch, fibre, dextrose syrup, biofuels, alcohol and other goods especially under moderate inputs and water deficit environments (Mehmood et al., 2008).

Ever increasing use of fertilizers and irrigation water, weed management has assumed significant importance in modern intensive farming, as the total loss of crop yield with increasing cost of cultivation causes a greater economic loss to the farmers (Aravadiya et al., 2012).

The extent of yield loss due to weeds primarily depends on the composition of weed flora, period of crop-weed-competition and its intensity. Weeds reduce the crop yields crop yield by competing with light, water, nutrients and carbon dioxide. Effect of weeds and weed management on uptake of nutrients and its effect on soil fertility status is scanty.

Therefore present experiment was planned to study the Influence of weed management practices on uptake of nutrients by kharif sorghum and its effect on soil fertility status.

\section{Materials and Methods}

A field experiment entitled "Influence of weed management practices on uptake of nutrientsby kharif sorghum [Sorghum bicolor (L.) Moench] and its effect on soil fertility status" was conducted at Agricultural Research Station, Hagari, Ballari during kharif 2018. Agricultural Research Station, Hagari, Ballari is located on $15^{\circ} 14^{\prime} \mathrm{N}$ latitude and $77^{\circ}$ 07' $\mathrm{E}$ longitude with an altitude of 414 meters above the mean sea level and is located in Northern Dry Zone of Karnataka (Zone-III).
The soil of the experimental field was clayey in texture. The soil available nutrient status showed medium availability of nitrogen (262.00 kg ha $\mathrm{kg}^{-1}$, medium in available phosphorus (39.25 $\left.\mathrm{kg} \mathrm{ha}^{-1}\right)$ and high in available potassium $\left(307.00 \mathrm{~kg} \mathrm{ha}^{-1}\right)$. The organic carbon content was low to medium $\left(5.1 \mathrm{~g} \mathrm{~kg}^{-1}\right)$. Twelve treatments comprising of weed management practices viz., $\mathrm{T}_{1}$ : Atrazine 50 WP @ $0.50 \mathrm{~kg}$ a.i. ha ${ }^{-1}$ as PE, $\mathrm{T}_{2}$ : Atrazine 50 WP @ $0.50 \mathrm{~kg}$ a.i. ha ${ }^{-1}$ as PE fb 1 IC at 40 DAS, $T_{3}$ : Pendimethalin 38.7 CS @0.6773 kg a.i. ha ${ }^{-1}$ as PE, T 4 : Pendimethalin $38.7 \mathrm{CS} @$ $0.6773 \mathrm{~kg}$ a.i. ha ${ }^{-1}$ as PE fb $1 \mathrm{IC}$ at 40 DAS, $\mathrm{T}_{5}$ : Atrazine $50 \mathrm{WP} @ 0.25 \mathrm{~kg}$ a.i. $\mathrm{ha}^{-1}+$ Pendimethalin 38.7 CS @ 0.3387 kg a.i. ha ${ }^{-1}$ (Tank mix) as PE, $\mathrm{T}_{6}$ : Atrazine 50 WP @ 0.25 kg a.i. ha ${ }^{-1}+$ Pendimethalin 38.7 CS @ 0.3387 $\mathrm{kg}$ a.i. $\mathrm{ha}^{-1}$ (Tank mix) as PE fb 1 IC at 40 DAS, T7: 2,4-D Ethyl Ester 38 EC @ $0.90 \mathrm{~kg}$ a.i. ha ${ }^{-1}$ as PoE at 30 DAS, T : 2,4-D Ethyl Ester 38 EC @ $0.90 \mathrm{~kg}$ a.i. ha ${ }^{-1}$ as PoE at 30 DAS fb 1 IC at 40 DAS, T9: Atrazine $50 \mathrm{WP}$ @ $0.50 \mathrm{~kg}$ a.i. $\mathrm{ha}^{-1}$ as PE fb 2,4-D Ethyl Ester 38 EC @ $0.90 \mathrm{~kg}$ a.i. ha ${ }^{-1}$ as PoE at 30 DAS, $\mathrm{T}_{10}$ : Hand weeding at $20 \mathrm{DAS}$ and $1 \mathrm{IC}$ at 40 DAS, $\mathrm{T}_{11}$ : Weed free (IC at $20 \& 40$ DAS and $\mathrm{HW}$ at $30 \mathrm{DAS}), \mathrm{T}_{12}$ : Weedy check were evaluated in randomized block design with three replications.

The composite soil samples from 0 to $15 \mathrm{~cm}$ depth were collected before planting and at harvest. Soils were air dried in shade, powdered and passed through $2 \mathrm{~mm}$ sieve and analysed for $\mathrm{pH}, \mathrm{EC}, \mathrm{OC}$, available $\mathrm{N}, \mathrm{P}_{2} \mathrm{O}_{5}$ and $\mathrm{K}_{2} \mathrm{O}_{5}$ by following the methods described by Jackson (1973).The seeds of CSH-25 @ $7.5 \mathrm{~kg} \mathrm{ha}^{-1}$ were sown at the spacing of $45 \mathrm{~cm}$ $\mathrm{X} 15 \mathrm{~cm}$ and the recommended package of practices were adopted for crop production.

The crop was harvested at its physiological maturity. The data was statistically analysed as per the procedure given by Panse and Sukhatme (1967). 


\section{Results and Discussion}

\section{Uptake of nutrients}

Significantly higher nitrogen, phosphorus and potassium uptake $\left(219,56.5\right.$ and $147.4 \mathrm{~kg} \mathrm{ha}^{-1}$, respectively) was recorded with preemergence application of atrazine 50 WP @ $0.50 \mathrm{~kg}$ a.i. ha $\mathrm{f}^{-1} \mathrm{fb}$ 2,4-D Ethyl Ester 38 EC @ $0.90 \mathrm{~kg}$ a.i. ha ${ }^{-1}$ as PoE at 30 DAS and it was on par with hand weeding at 20 DAS and 1 IC at 40 DAS $\left(215,55.2\right.$, and $145.7 \mathrm{~kg} \mathrm{ha}^{-1}$, respectively) and 2,4-D Ethyl Ester 38 EC @ $0.90 \mathrm{~kg}$ a.i. $\mathrm{ha}^{-1}$ as PoE at $30 \mathrm{DAS}$ fb $1 \mathrm{IC}$ at 40 DAS (211, 54.9, and $143.7 \mathrm{~kg} \mathrm{ha}^{-1}$, respectively) (Table 1). Whereas, significantly lower uptake of nitrogen, phosphorus and potassium was observed with weedy check $\left(129,35.7\right.$ and $95.4 \mathrm{~kg} \mathrm{ha}^{-1}$, respectively) as compared to other treatments (Table 2).

This is due to lower crop weed competition results in better dry matter production in crop this ultimately results in higher uptake of nutrients. Whereas, the lowest uptake of nitrogen, phosphorus and potassium by maize crop was recorded in unweeded control as a result of weed competition resulting in lower dry matter production.

These results corroborate the results of Priya and Kubsad (2013) in sorghum and also by Sreenivas and Satyanarayana (1994).

\section{Soil fertility status}

Nitrogen, phosphorus and potassium availability in the soil after harvest of kharif sorghum crop was significantly influenced by different weed management practices (Table 2).

Significantly higher available nitrogen, phosphorus and potassium in the soil were recorded with weed free (IC at $20 \& 40$ DAS and $\mathrm{HW}$ at 30 DAS) $(194,47.6$, and $219.9 \mathrm{~kg}$ $\mathrm{ha}^{-1}$, respectively). Among other weed management treatments, sequential application of pre-emergence herbicide atrazine 50 WP @ $0.50 \mathrm{~kg}$ a.i. ha ${ }^{-1} \mathrm{fb}$ 2,4-D Ethyl Ester 38 EC @ $0.90 \mathrm{~kg}$ a.i. $\mathrm{ha}^{-1}$ as PoE at 30 DAS recorded significantly higher availability of nitrogen, phosphorus and potassium in the soil (177, 42.2 , and $213.8 \mathrm{~kg} \mathrm{ha}^{-1}$, respectively) and it was on par with hand weeding at 20 DAS and $1 \mathrm{IC}$ at 40 DAS $\left(173,41.7\right.$, and $212.1 \mathrm{~kg} \mathrm{ha}^{-1}$, respectively) and 2,4-D Ethyl Ester 38 EC @ $0.90 \mathrm{~kg}$ a.i. $\mathrm{ha}^{-1}$ as PoE at $30 \mathrm{DAS}$ fb $1 \mathrm{IC}$ at 40 DAS (170, 39.9, and $211.9 \mathrm{~kg} \mathrm{ha}^{-1}$, respectively).

Significantly lower content of available nitrogen, phosphorus and potassium was noticed with weedy check (129, 26.9 and $152.0 \mathrm{~kg} \mathrm{ha}^{-1}$, respectively) compared to other treatments. These results are in corroboration with findings of Verma et al., (2017).

\section{Residual effect of herbicides on succeeding chickpea crop}

The germination percentage, plant height and number of branches of chickpea were recorded at 45 DAS and found that, treatments did not differ significantly (Table 3 ). The sorghum chickpea is the prominent sequence in the experimental area.

Hence, the residual effects of these treatments were studied on chickpea by bioassay studies (germination test) and the crop was examined for its growth parameters like plant height and branches in main field.

The data showed that non-significant differences between chemical weed management practices and non-chemical treated plots (hand weeding, weed free and weedy check) indicating no adverse effect of applied herbicides on succeeding crop and confirmed no residual effect of the herbicides tried in the experiment. 
Table.1 Nitrogen, phosphorus and potassium uptake by kharif sorghum as influenced by different weed management practices at harvest

\begin{tabular}{|c|c|c|c|}
\hline \multirow[t]{2}{*}{ Treatments } & \multicolumn{3}{|c|}{ Uptake of nutrients $\left(\mathrm{kg} \mathrm{ha}^{-1}\right)$} \\
\hline & Nitrogen & $\begin{array}{l}\text { Phosphorus } \\
\qquad\left(\mathbf{P}_{2} \mathbf{O}_{5}\right)\end{array}$ & $\begin{array}{l}\text { Potassium } \\
\quad\left(\mathbf{K}_{2} \mathbf{O}\right)\end{array}$ \\
\hline $\mathrm{T}_{1}:$ Atrazine 50 WP @ $0.50 \mathrm{~kg}$ a.i. ha ${ }^{-1}$ as PE & 181 & 45.0 & 120.4 \\
\hline$T_{2}$ : Atrazine 50 WP @ $0.50 \mathrm{~kg}$ a.i. $\mathrm{ha}^{-1}$ as PE fb one IC at 40 DAS & 204 & 49.0 & 138.2 \\
\hline $\mathrm{T}_{3}$ : Pendimethalin $38.7 \mathrm{CS} @ 0.6773 \mathrm{~kg}$ a.i. $\mathrm{ha}^{-1}$ as PE & 139 & 41.2 & 101.5 \\
\hline $\mathrm{T}_{4}:$ Pendimethalin $38.7 \mathrm{CS} @ 0.6773 \mathrm{~kg}$ a.i. ha ${ }^{-1}$ as PE fb one IC at 40 DAS & 170 & 42.9 & 118.6 \\
\hline $\begin{array}{l}\text { T5 : Atrazine } 50 \text { WP @ } 0.25 \text { kg a.i. ha }{ }^{-1}+\text { Pendimethalin } 38.7 \text { CS @ } 0.3387 \text { kg a.i. ha }{ }^{-1} \text { (Tank mix) as } \\
\text { PE }\end{array}$ & 158 & 41.4 & 109.1 \\
\hline $\begin{array}{l}\text { T6: Atrazine } 50 \text { WP @ } 0.25 \text { kg a.i. ha }{ }^{-1}+\text { Pendimethalin } 38.7 \text { CS @ } 0.3387 \text { kg a.i. ha }{ }^{-1} \text { (Tank mix) as } \\
\text { PE fb one IC at } 40 \text { DAS }\end{array}$ & 169 & 46.1 & 116.9 \\
\hline $\mathrm{T}_{7}:$ 2,4-D Ethyl Ester 38 EC @ 0.90 kg a.i. ha ${ }^{-1}$ as PoE at 30 DAS & 191 & 48.4 & 125.3 \\
\hline $\mathrm{T}_{8}: 2,4-\mathrm{D}$ Ethyl Ester $38 \mathrm{EC} @ 0.90 \mathrm{~kg}$ a.i.ha ${ }^{-1}$ as PoE at $30 \mathrm{DAS}$ fb one IC at 40 DAS & 211 & 54.9 & 143.7 \\
\hline $\begin{array}{l}\text { T9: Atrazine } 50 \text { WP @ } 0.50 \text { kg a.i. ha }{ }^{-1} \text { as PE fb 2,4-D Ethyl Ester } 38 \text { EC @ } 0.90 \text { kga.i. ha }{ }^{-1} \text { as PoE } \\
\text { at } \\
\text { DAS }\end{array}$ & 219 & 56.5 & 147.4 \\
\hline$T_{10}:$ HW at 20 DAS and one IC at 40 DAS & 215 & 55.2 & 145.7 \\
\hline$T_{11}:$ Weed free (IC at $20 \& 40$ DAS and HW at 30 DAS) & 235 & 61.9 & 159.4 \\
\hline $\mathbf{T}_{12}:$ Weedy check & 129 & 35.7 & 95.4 \\
\hline S.Em \pm & 2.8 & 0.9 & 1.7 \\
\hline C.D. $(P=0.05)$ & 8.1 & 2.7 & 5.0 \\
\hline
\end{tabular}

\section{Note:}

WP: Wettable powder

IC: Inter cultivation
PE: Pre-emergence

DAS: Days after sowing fb: Followed by

CS: Capsulated suspension
HW: Hand weeding

EC: Emulsified concentrate
PoE: Post emergence 
Table.2 Effect of different weed management practices on available NPK content in soil after harvest of kharif sorghum

\begin{tabular}{|c|c|c|c|}
\hline \multirow[t]{2}{*}{ Treatments } & \multicolumn{3}{|c|}{ Available nutrients $\left(\mathrm{kg} \mathrm{ha}^{-1}\right)$} \\
\hline & Nitrogen & $\begin{array}{c}\text { Phosphorus } \\
\left(\mathbf{P}_{2} \mathbf{O}_{5}\right)\end{array}$ & $\begin{array}{l}\text { Potassium } \\
\left(\mathbf{K}_{2} \mathbf{O}\right)\end{array}$ \\
\hline$T_{1}$ : Atrazine 50 WP @ 0.50 kg a.i. ha ${ }^{-1}$ as PE & 153 & 35.5 & 191.1 \\
\hline $\mathrm{T}_{2}$ : Atrazine 50 WP @ $0.50 \mathrm{~kg}$ a.i. $\mathrm{ha}^{-1}$ as PE fb one IC at $40 \mathrm{DAS}$ & 159 & 37.5 & 205.6 \\
\hline$T_{3}:$ Pendimethalin 38.7 CS @ $0.6773 \mathrm{~kg}$ a.i. ha ${ }^{-1}$ as PE & 134 & 27.1 & 170.3 \\
\hline $\mathrm{T}_{4}$ : Pendimethalin $38.7 \mathrm{CS} @ 0.6773 \mathrm{~kg}$ a.i. $\mathrm{ha}^{-1}$ as PE fb one IC at $40 \mathrm{DAS}$ & 145 & 31.3 & 179.0 \\
\hline $\mathrm{T}_{5}$ : Atrazine 50 WP @ $0.25 \mathrm{~kg}$ a.i. ha ${ }^{-1}+$ Pendimethalin $38.7 \mathrm{CS} @ 0.3387 \mathrm{~kg}$ a.i. ha ${ }^{-1}$ (Tank mix) as PE & 141 & 30.0 & 174.3 \\
\hline $\begin{array}{l}\mathrm{T}_{6} \text { : Atrazine } 50 \text { WP @ } 0.25 \mathrm{~kg} \text { a.i. } \mathrm{ha}^{-1}+\text { Pendimethalin } 38.7 \mathrm{CS} @ 0.3387 \mathrm{kga.i}^{-} \mathrm{ha}^{-1} \text { (Tank mix) as PE fb } \\
\text { one IC at } 40 \text { DAS }\end{array}$ & 148 & 33.9 & 179.9 \\
\hline $\mathrm{T}_{7}:$ 2,4-D Ethyl Ester $38 \mathrm{EC} @ 0.90 \mathrm{~kg}$ a.i. ha ${ }^{-1}$ as PoE at $30 \mathrm{DAS}$ & 155 & 37.1 & 194.4 \\
\hline $\mathrm{T}_{8}:$ 2,4-D Ethyl Ester $38 \mathrm{EC} @ 0.90 \mathrm{~kg}$ a.i.ha ${ }^{-1}$ as PoE at $30 \mathrm{DAS}$ fb one IC at 40 DAS & 170 & 39.9 & 211.9 \\
\hline $\begin{array}{l}\text { T}_{9} \text { : Atrazine } 50 \text { WP @ } 0.50 \mathrm{~kg} \text { a.i. ha }{ }^{-1} \text { as PE fb 2,4-D Ethyl Ester } 38 \text { EC @ } 0.90 \mathrm{kga.i.} \mathrm{ha}^{-1} \text { as PoE at } 30 \\
\text { DAS }\end{array}$ & 177 & 42.2 & 213.8 \\
\hline$T_{10}: H W$ at 20 DAS and one IC at 40 DAS & 173 & 41.7 & 212.1 \\
\hline$T_{11}:$ Weed free (IC at $20 \& 40$ DAS and $\mathrm{HW}$ at $\left.30 \mathrm{DAS}\right)$ & 194 & 47.6 & 219.9 \\
\hline $\mathbf{T}_{12}:$ Weedy check & 129 & 26.9 & 152.0 \\
\hline S.Em \pm & 2.6 & 1.5 & 1.3 \\
\hline C.D. $(P=0.05)$ & 7.6 & 4.4 & 3.8 \\
\hline
\end{tabular}

\section{Note:}

WP: Wettable powder

IC: Inter cultivation
PE: Pre-emergence

DAS: Days after sowing fb: Followed by

CS: Capsulated suspension
HW: Hand weeding

PoE: Post emergence
EC: Emulsified concentrate 
Table.3 Germination percentage, plant height and number of branches per plant of succeeding chickpea at 45 DAS as influenced by different weed management practices in kharif sorghum

\begin{tabular}{|c|c|c|c|}
\hline Treatments & $\begin{array}{l}\text { Germination } \\
(\%)\end{array}$ & $\begin{array}{l}\text { Plant height } \\
\quad(\mathrm{cm})\end{array}$ & $\begin{array}{l}\text { Number of } \\
\text { branches plant }^{-1}\end{array}$ \\
\hline $\mathrm{T}_{1}$ : Atrazine 50 WP @ $0.50 \mathrm{~kg}$ a.i. ha ${ }^{-1}$ as PE & 88 & 18.81 & 3.7 \\
\hline $\mathrm{T}_{2}:$ Atrazine 50 WP @ $0.50 \mathrm{~kg}$ a.i. $\mathrm{ha}^{-1}$ as PE fb one IC at $40 \mathrm{DAS}$ & 86 & 18.33 & 3.3 \\
\hline $\mathrm{T}_{3}:$ Pendimethalin $38.7 \mathrm{CS} @ 0.6773 \mathrm{~kg}$ a.i. ha ${ }^{-1}$ as PE & 87 & 18.00 & 3.5 \\
\hline $\mathrm{T}_{4}:$ Pendimethalin $38.7 \mathrm{CS} @ 0.6773 \mathrm{~kg}$ a.i. ha ${ }^{-1}$ as PE fb one IC at 40 DAS & 87 & 18.19 & 3.9 \\
\hline $\begin{array}{l}\mathrm{T}_{5} \text { : Atrazine } 50 \text { WP @ } 0.25 \mathrm{~kg} \text { a.i. ha }{ }^{-1}+\text { Pendimethalin } 38.7 \mathrm{CS} @ 0.3387 \mathrm{~kg} \text { a.i. ha }{ }^{-1}(\text { Tank } \\
\text { mix) as PE }\end{array}$ & 87 & 19.58 & 3.9 \\
\hline $\begin{array}{l}\mathrm{T}_{6} \text { : Atrazine } 50 \text { WP @ } 0.25 \mathrm{~kg} \text { a.i. ha }{ }^{-1}+\text { Pendimethalin } 38.7 \mathrm{CS} @ 0.3387 \mathrm{kga.i}^{-1} \mathrm{ha}^{-1} \text { (Tank } \text { mix) as PE fb one IC at } 40 \text { DAS }\end{array}$ & 88 & 18.33 & 3.3 \\
\hline $\mathrm{T}_{7}:$ 2,4-D Ethyl Ester 38 EC @ 0.90 kg a.i. ha ${ }^{-1}$ as PoE at 30 DAS & 88 & 18.38 & 3.4 \\
\hline T $:$ 2,4-D Ethyl Ester 38 EC @ $0.90 \mathrm{~kg}$ a.i.ha $^{-1}$ as PoE at 30 DAS fb one IC at 40 DAS & 87 & 18.71 & 3.8 \\
\hline $\begin{array}{l}\mathrm{T}_{9} \text { : Atrazine } 50 \text { WP @ } 0.50 \mathrm{~kg} \text { a.i. } \mathrm{ha}^{-1} \text { as PE fb 2,4-D Ethyl Ester } 38 \text { EC @ } 0.90 \mathrm{kga.i}^{-\mathrm{ha}^{-1}} \\
\text { as PoE at } 30 \text { DAS }\end{array}$ & 88 & 19.38 & 3.7 \\
\hline $\mathrm{T}_{10}: \mathrm{HW}$ at $20 \mathrm{DAS}$ and one IC at 40 DAS & 90 & 19.05 & 3.6 \\
\hline $\mathrm{T}_{11}:$ Weed free (IC at $20 \& 40 \mathrm{DAS}$ and $\mathrm{HW}$ at $\left.30 \mathrm{DAS}\right)$ & 90 & 20.67 & 4.0 \\
\hline $\mathbf{T}_{12}:$ Weedy check & 89 & 18.00 & 3.6 \\
\hline S.Em \pm & 0.9 & 2.0 & 0.7 \\
\hline C.D. $(\mathbf{P}=0.05)$ & NS & NS & NS \\
\hline
\end{tabular}

\section{Note:}

WP: Wettable powder

IC: Inter cultivation
PE: Pre-emergence

DAS: Days after sowing fb: Followed by

CS: Capsulated suspension
HW: Hand weeding

PoE: Post emergence 
Jayakumar et al., (2003) obtained similar results in sorghum. The results of the investigation revealed that significantly higher uptake of nutrients by kharif sorghum and available nitrogen, phosphorus and potassium in soil were recorded with Pre-emergence application of atrazine $50 \mathrm{WP} @ 0.50 \mathrm{~kg}$ a.i. $\mathrm{ha}^{-1} \mathrm{fb}$ 2,4-D Ethyl Ester 38 EC @ $0.90 \mathrm{~kg}$ a.i. $\mathrm{ha}^{-1}$ as PoE at 30days after sowing and no significance phytotoxic effect was recorded in succeeding chickpea crop on various growth attributes.

\section{References}

Arvadiya, L. K., Raju, V.C., Patel, T.U. and Aravadiya, M.K., 2012, Influence of plant population and weed management on weed flora and productivity of sweet corn (zea mays L.). Indian J. Agron., 57(2): 162-167.

Jackson, M. L., 1973, Soil Chemical Analysis. Prentice hall of India (Pvt.), New Delhi, pp. 67-214.
Jayakumar, R., Ali, A. M. and Subramanian, S., 2003. Studies on residues of herbicides applied in cotton (Gossypium hirsutum L.) by bioassay techniques. Pesticide Res. J., 14(3): 263-267.

Panse, V. G. and Sukhatme, P. U., 1967, Statistical Methods for Agricultural Workers.ICAR, New Delhi.

Priya, H. R. and Kubsad, V. S., 2013. Integrated weed management in rainy season sorghum (Sorghum bicolor L.). Indian J. Agron., 58(4): 548-553.

Verma, B. R., Virdia, H. M. and Dinesh, K., 2017. Effect of Integrated weed management on yield, quality and economics of summer sorghum (Sorghum bicolor L.). Intl. J. Curr. Microbiol. App. Sci., 6(8): 1630-1636.

Sreenivas, G. and Satyanarayana, V., 1994.Nutrient removal by weeds and maize (Zea mays L.).Indian J. Agron., 41(1): 160-162.

\section{How to cite this article:}

Vinayaka, S. S., D. Krishnamurthy, A. S. Channabasavanna, Y. M. Ramesha and Manjunath Bhanuvalli. 2019. Influence of Weed Management Practices on Uptake of Nutrients by Kharif Sorghum [Sorghum bicolor (L.) Moench] and its Effect on Soil Fertility Status. Int.J.Curr.Microbiol.App.Sci. 8(10): 261-267. doi: https://doi.org/10.20546/ijcmas.2019.810.027 